\title{
Peptostreptococcus tetradius: a new pathogenous for the acute ocular infections?
}

\author{
Franco Giardini', Erika Savio', Romolo Protti ${ }^{2}$, Cristina Pollino', Tullio Albanesi', \\ Gabriella Furfaro', Giuseppe Grandi' \\ I Laboratorio Analisi ,Ospedale Oftalmico, Torino \\ 2 Traumatologia Oftalmologica, Ospedale Oftalmico, Torino
}

Key Words: Ocular infections, Severe prognosis in ocular infections, Endophthalmitis, Peptostreptococcus tetradius.

\section{Peptostreptococcus tetradius: un nuovo patogeno di infezioni oculari a prognosi severa?}

\section{SUMMARY}

In our Ophthalmologic Hospital (Turin ,Italy) was isolated from some years a new pathogenous, in the our experience, expecially in the ocular infections and endophthalmitis.

The our casistic of the genus Peptostreptococcus in five years is of 23 cases.

In this work are described also 2 clinic cases with final discussion.

We have also examinated the sensibility of this pathogenous versus all the antibiotics for topical ocular use.

\section{INTRODUZIONE}

Il genere Peptostreptococcus comprende cocchi Gram + che si presentano al microscopio sotto forma di tetradi, più raramente a paia o a brevi catenelle, con cocchi piuttosto grossi.

Sino a pochissimi anni fa' era chiamato Gaffkja ed era considerato un banale saprofita.

La casistica degli isolamenti colturali da infezioni oculari presso l'Ospedale Oftalmico di Torino comprende ben 23 casi di Peptostreptococcus tetradius dal 2007 a tutto il 2012. Per il nostro Ospedale si tratta di un nuovo patogeno oculare (4) poiché nei 10 anni precedenti erano stati isolati, dagli stessi operatori, solo 3 ceppi.

\section{MATERIALI E METODI}

I pazienti da noi arruolati erano in buona parte affetti da patologie oculari a prognosi severa, e per la metà (11 su 23) erano diabetici. Cinque erano i casi di endoftalmite (pari al $21.6 \%$ ), quattro i casi di ascesso (pari al 17.3\%) mentre il totale dei casi di pazienti interessati da infezioni a prognosi severa era del $52.2 \%$.

Due pazienti erano ricoverati (uno aveva subito poco tempo prima l'intervento di cataratta (5)) mentre tutti gli altri erano esterni ed erano afferiti al nostro Pronto Soccorso.

I terreni adottati per l'isolamento erano l'Agar Cioccolato in aerobiosi, l'agar Cioccolato Haemophilus in capnofilia, il brodo Cuore Cervello ed eventualmente i flaconi Bactec Ped Plus e Bactec Mycosis (BD) per l'isolamento da pazienti con infezioni oculari molto serie e con in corso pesanti trattamenti antibiotici, nonché quanto il nostro protocollo prevede per l'isolamento degli anaerobi $(2,4)$.

Tutto questo dopo una colorazione Gram preliminare.

I campioni di Vitreo e Acqueo erano trasportati presso la Microbiologia del nostro Laboratorio mediante contenitori Port-a-Cult (BD).

In particolare desideriamo considerare due casi su tutti:

\section{CASO A}

R.T. donna, 66 anni, diabetica, intervento faco + IOL.

In $3^{\circ}$ giornata manifesta dolore e forte arrossamento.

In $7^{\circ}$ giornata vitrectomia e occhio riempito con silicone, prelievo di vitreo e acqueo.

In $8^{\circ}$ giornata sviluppo di Peptostreptococcus tetradius multiresistente e di Bacillaceae anch'esse multiresistenti (3).

In $9^{\circ}$ giornata l'antibiogramma rivela una sensibilità in vitro del $P$. tetradius solo a Levofloxacina, Cloramfenicolo, Moxifloxacina, Tetraciclina, Acido Fusidico.

La rapidità dello sviluppo colturale (18 h) dai flaconi Bactec ha fornito all'oculista indicazioni terapeutiche con immediatezza e soprattutto mirate verso l'endoftalmite (1).

Grazie ad una terapia con collirii topici rinforzati (Levofloxacina e Cloramfenicolo) più una terapia sistemica con Cefalosporine di III generazione e Netilmicina queste cure rapide e mirate hanno

\section{Corresponding author: Franco Giardini}

Via Avigliana 72; 10093 Leumann (TO)

Tel. 3477076053

E-mail: franco.giardini@libero.it 
consentito di salvare l'integrità anatomica dell'occhio (4).

\section{CASO B}

L.B. uomo, 28 anni, portatore di LAC, in Pronto Soccorso rilevata ulcera corneale marcata il cui esordio pare risalire a $6 \mathrm{gg}$. prima. Il paziente si presenta con una autoterapia antibiotica di Tobral + Augmentin (consigliato dal farmacista). Viene prescritto Exocin locale.

In $2^{\circ}$ giornata dal ricovero isolamento di Peptostreptococcus tetradius con un antibiogramma che rivela invece sensibilità in vitro solo per Cloramfenicolo, Netilmicina, Levofloxacina, Norfloxacina, Lomefloxacina. Con una terapia di Nettacin, Okacin, Colbiocin ogni ora, + Rocefin sistemico (1).

In $7^{\circ}$ giornata l'ulcera corneale centrale si riduce moltissimo.

In $9^{\circ}$ giornata il paziente viene dimesso con l'ulcera di dimensioni ridottissime.

\section{DISCUSSIONE}

Anzitutto ci preme sottolineare la vera e propria "esplosione" di casi di infezione oculare ascrivibili a questo schizomicete in questi ultimi 5 anni della nostra casistica (2). Questo deve mettere all'erta gli oculisti ed i nostri colleghi microbiologi che si interessano di batteriologia oculare.

Circa poi le patologie oculari da cui abbiamo isolato questi schizomiceti notiamo che sostanzialmente le patologie severe e le congiuntiviti si spartiscono al $50 \%$ i casi considerati.

Ciò significa che si tratta di un batterio che a livello oculare può causare una notevole percentuale di casi la cui evoluzione può determinare patologie molto serie.

Quanto allo spettro di sensibilità agli antibatterici per uso topico oculare notiamo che il Cloramfenicolo e la Netilmicina sono quelli che in vitro hanno un'azione più efficace, unitamente ad alcuni fluorochinoloni come la Moxifloxacina, la Levofloxacina, la Ciprofloxacina.

Scarsissima l'efficacia di Vancomicina (spesso acriticamente messa nei protocolli verso le endoftalmiti) (1, 5) nonchè di Tobramicina e di Neomicina.

\section{CONCLUSIONI}

$\mathrm{Ci}$ pare molto importante segnalare questo "nuovo" patogeno che si rivela interessante a livello oculare anche (e forse soprattutto) per la sua aggressività.

La nostra casistica in materia (2), che sarebbe utile confrontare con quella di altri centri di Oftalmologia, ci conferma la assoluta necessità di effettuare immediatamente un esame colturale in tutti i casi di infezione oculare di una certa gravità e di considerare con attenzione l'eventuale isolamento di Peptostreptococcus tetradius da infezioni oculari.

La rapidità dello sviluppo colturale dei flaconi Bactec e le loro peculiarità biochimiche hanno consentito di fornire all'oculista indicazioni terapeutiche mirate verso le infezioni oculari gravi che hanno manifestato caratteri di aggressività e di repentina evoluzione (4).

Con il supporto degli antibiogrammi è stata poi attuata una terapia multimolecolare sia topica che sistemica.

Tabella I. Resistenze e sensibilità in vitro nel primo caso clinico

\begin{tabular}{|l|c|c|}
\cline { 2 - 3 } \multicolumn{1}{c|}{} & $\begin{array}{c}\text { ATB sul ceppo di } \\
\text { BACILLACEAE }\end{array}$ & $\begin{array}{c}\text { ATB su } \\
\text { Peptostreptococcustetradius }\end{array}$ \\
\hline AMPICILLINA & $\mathrm{S}$ & $\mathrm{R}$ \\
\hline CLORAMFENICOLO & $\mathrm{S}$ & $\mathrm{S}$ \\
\hline TETRACICLINA & $\mathrm{R}$ & $\mathrm{S}$ \\
\hline NEOMICINA & $\mathrm{R}$ & $\mathrm{R}$ \\
\hline NETILMICINA & $\mathrm{R}$ & $\mathrm{R}$ \\
\hline AMIKACINA & $\mathrm{R}$ & $\mathrm{R}$ \\
\hline GENTAMICINA & $\mathrm{R}$ & $\mathrm{R}$ \\
\hline TOBRAMICINA & $\mathrm{R}$ & $\mathrm{R}$ \\
\hline VANCOMICINA & $\mathrm{R}$ & $\mathrm{R}$ \\
\hline CIPROFLOXACINA & $\mathrm{R}$ & $\mathrm{R}$ \\
\hline MOXIFLOXACINA & $\mathrm{S}$ & $\mathrm{S}$ \\
\hline OFLOXACINA & $\mathrm{S}$ & $\mathrm{R}$ \\
\hline LOMEFLOXACINA & $\mathrm{R}$ & $\mathrm{R}$ \\
\hline LEVOFLOXACINA & $\mathrm{R}$ & $\mathrm{S}$ \\
\hline NORFLOXACINA & $\mathrm{R}$ & $\mathrm{R}$ \\
\hline AC.FUSIDICO & $\mathrm{R}$ & $\mathrm{S}$ \\
\hline
\end{tabular}




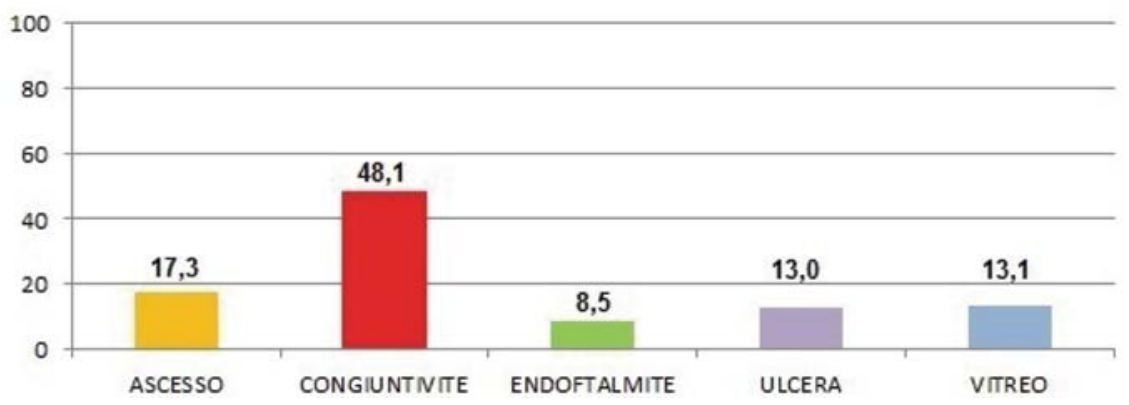

Figura I. Percentuali Peptostreptococcus spp per patologia

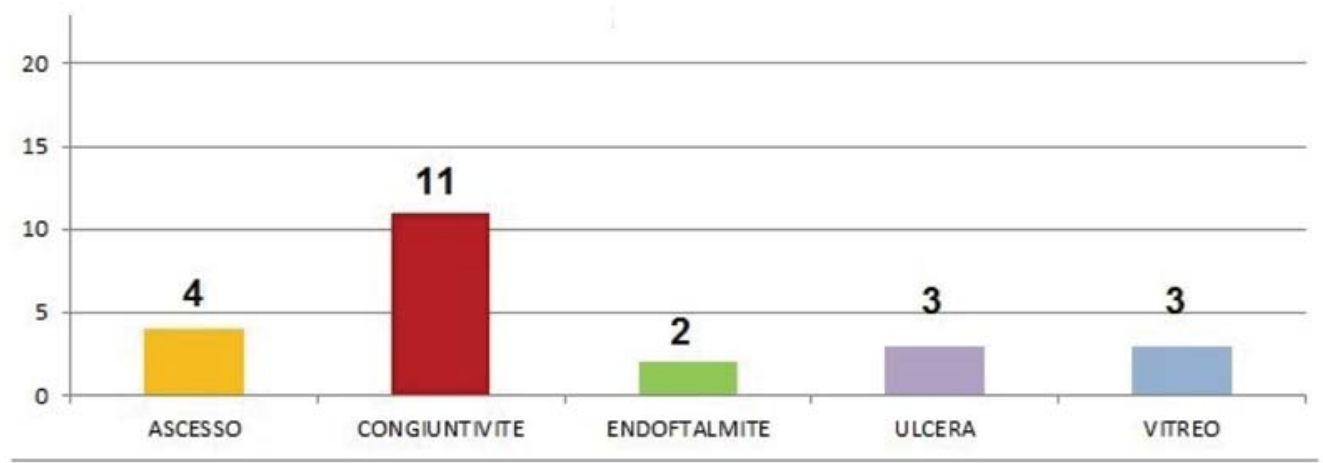

Figura II. I 23 casi totali suddivisi per patologia

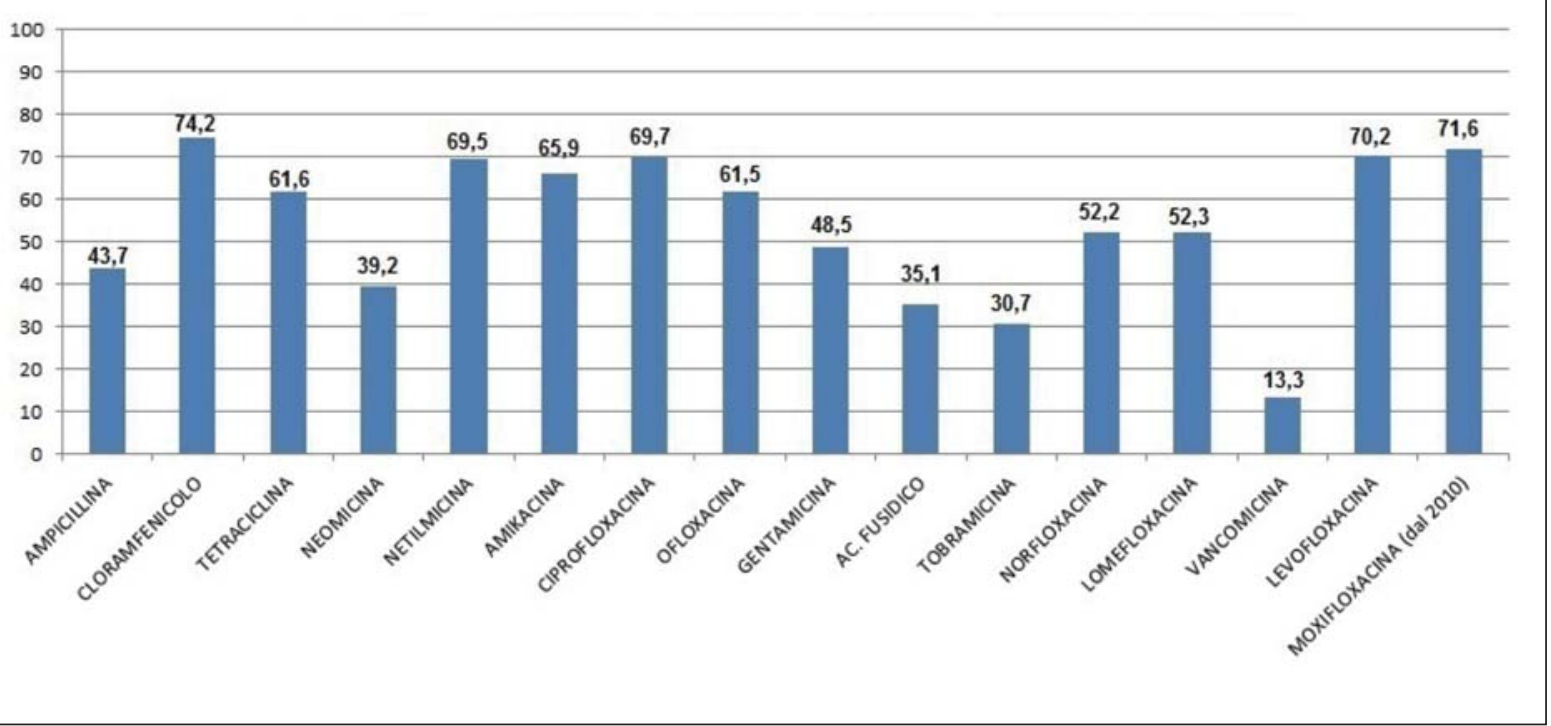

Figura III. Percentuali di sensibilità agli antibiotici di Peptostreptococcus spp 


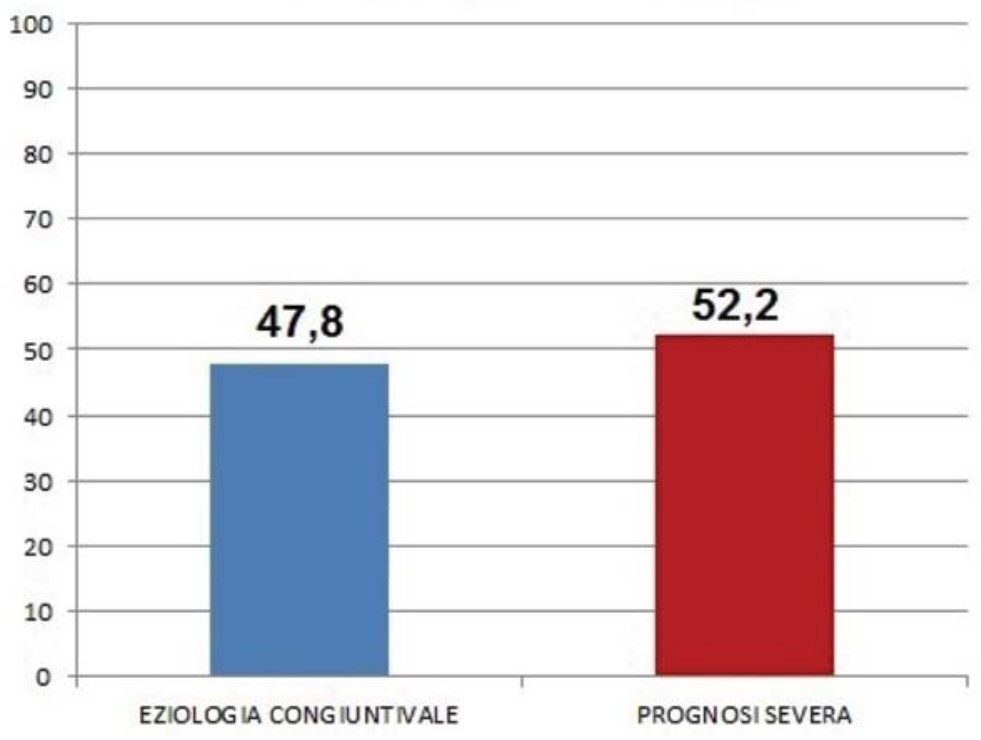

Figura IV. Percentuale Peptostreptococcus spp

\section{BIBLIOGRAFIA}

1. Forster R, Abbott RL, Gelender H. Management of infectious endolphthalmitis. Ophthalmology. 1980; 87: 313-9.

2. Grandi G, Grignolo FM, Giardini F. Manuale di Microbiologia Oculare. Medical Systems-Genova2011.

3. Miller J, Scott IU, Flynn HW Jr, Smiddy WE.
Endophthalmitis caused by Bacillus species. Am J Ophthalmology. 2008 May; 145 (5): 883-8.

4. Nuzzi R, Tonini L, Consolandi G, Pollino C, Giardini F. Endoftalmiti: clinica, indagini di laboratorio e terapia. Minerva Oftalmologica Settembre 2009; 51 (3).

5. Taban M, Behrens A, Newcomb RL, Nobe MY. Acute endophthalmitis following cataract surgery. Arch Ophthalmol. 2005; 123: 613-20. 